\title{
The Role of Plant Mediator as a Gene Regulator to Tackle Climate Change: A Review
}

\author{
D. L. Wathugala \\ Dept. of Crop Science, Faculty of Agriculture, \\ University of Ruhuna, Mapalana, Kamburupitiya, Sri Lanka \\ Tele: (94) 71826 5218; Fax: (94) 41229 2384; E-mail:wgdlakmini@crop.ruh.ac.lk
}

\begin{abstract}
The current rate of climate change predicts that plants will become subject to increasing extremes of environmental stress. Rapid population increase in developing countries also demand higher yield from crop production, often from sub-optimal agricultural areas. Genetic engineering can help to meet these needs through the development of crops with greater stress tolerance. Mediator is transcriptional co-activators which convey DNA bound transcriptional regulators and enhancers to the general RNA polymerase II transcription machinery and mediator genes are recently identified in plants. So far it has been showed that their great involvement in regulation of plant stress tolerance by controlling transcription of stress genes. Therefore, Mediator genes will be a good source in future molecular biology researches on manipulation of crop plants with multiple stress tolerance.
\end{abstract}

KEYWORDS: Mediator genes, Environmental stress, Stress tolerance

\section{Introduction}

Developing stress tolerant crops to cope with the rapid environmental degradation that is occurring is an absolute requirement in order to provide enough food for growing population. One of the basic genetic engineering approaches currently being used to improve crop stress tolerance is generation of transgenic plants by introducing novel genes into the genome of agriculturally important crops or altering the expression of existing genes. Understanding stress response signaling pathways is the prime requirement to manipulate stress tolerance of crop plants by this approach. Recent studies have made progress in the definition of various genetic protective responses to stress stimuli using the model plant species Arabidopsis thaliana. However, almost all these studies focused on only one stress condition. Therefore, finding ways to produce multiple stress tolerant crops are important. Mediator is the evolutionary conserved multi-protein complex that binds RNA polymerase II and controls transcription of genes (Flanagan et al., 1991). The possible mechanism suggested by Chadick and Asturias (2005), Bjorklund and 
Gustafsson (2005), and Hahn (2004) for transcriptional activation by mediator was that the gene specific activators (transcription factors) recruits mediator to the transcription initiation site. Then, general transcription factors involved in gene transcription interact with the DNA-mediator complex to form a platform to bind RNA polymerase II.

Therefore, the focus of this review is to provide information on the plant mediator to generate interest among researches as a possible route to improve crop tolerance to environmental stresses. It first provides a short overview of past research that discusses changes of gene expression in response to environmental stresses. Next it describes transcription factors that regulate stress responses. The role of plant mediators in stress gene expression is then discussed. Then the paper concludes by summarizing the importance of future research needs of plant mediator as a possible route to improve crop tolerance to multiple environmental stresses.

\section{Changes in Gene Expression in Response to Environmental Stresses}

Stress responses are initiated when plants recognize stress at the cellular level, followed by generation of second messengers such as calcium and ROS (Reactive Oxygen Species). These then trigger activation of various signal transduction pathways (Fowler and Thomashow, 2002; Knight and Knight, 2001) which transmit information and activate stress inducible gene expression. Microarrays in different plant species show a significant overlap in the types of genes whose expression is altered upon exposure to different stresses (Schenk et al., 2000; Chen et al., 2002; Seki et al., 2002). For example, Seki et al. (2002) report that 22 genes (out of 7000 from Arabidopsis) form part of a common transcriptional response to cold, drought and salt induced stresses.

Another microarray analysis, by Chen et al. (2002) using 402 Arabidopsis transcription factors, showed extensive overlaps in expression of similar genes under different stresses. Nawrath et al. (2002) also showed the induction of a number of genes in common between pathogen attack and UV irradiance. The encoded proteins function either to protect cells from stress damage directly, or are involved in the generation of regulatory molecules like abscicic acid (ABA) ethylene and salicylic acid (SA) which themselves further regulate gene expression (Shinozaki et al., 2003). The signaling pathways of stress induced gene expression are not yet completely understood, though the transcriptional activation of some stress induced genes has been well studied (Xiong et al., 2002).

\section{Transcription Factors Regulate Stress Responses}

Transcription factors are genetic switches which fine tunes all responses of plants by activating promoters of inducible genes. Many transcription factor genes are found among the list of genes up and down regulated in response to environmental 
stresses (Seki et al., 2001; Kreps et al., 2002; Seki et al., 2002; Chen et al., 2002; Shinozaki et al., 2003).

Stress-inducible expression of transcription factors include members of the AP2/EREBP (apetala2/ethylene-responsive element binding proteins) family, the zinc-finger family, the WRKY family ${ }^{1}$, the MYB (myeloblastosis) family, the basic helix-loop-helix (bHLH) family, the basic-domain leucine zipper (bZIP) family, the NAC family ${ }^{2}$, and the homeodomain transcription factor family (Shinozaki et al., 2003). The AP2/EREBP transcription factor family, bZIP family and WRKY family proteins are reported as major players in the regulation of gene expression in response to environmental stresses (Singh et al., 2002). Furthermore, significant improvement of stress tolerance was observed upon over-expression of individual transcription factors from these families. As examples, over-expression of $C B F 1 / D R E B 1 B$ and DREB1A (sub families of AP2/EREBP family) in Arabidopsis greatly increases the plant's resistance to cold, drought and salt (Jaglo-Ottosen et al., 1998; Kasuga et al., 1999).

Both induction and repression of specific transcription factors are required to fine tune the plant to various environmental stresses. For example, MYB transcription factors are associated with responses to UV light and pathogen infection (Rushton and Somssich, 1998; Singh et al., 2002), though Jin and colleagues (2000) report that AtMYB4 represses target genes under UV-B irradiance, and the overexpression of AtMYB4 reduces the UV-B tolerance of Arabidopsis.

AP2/EREBP comprises the biggest transcription factor family in Arabidopsis, with 45 members (Riechmann et al., 2000). These transcription factors are linked with a wide range of stress responses. One subfamily of AP2/EREBP, known as CBF/DREB1 (C-repeat binding factor/drought responsive element binding factor1), bind a cis-element known as the CRT/DRE (C-repeat/drought responsive element), common to the promoters of COR (Cold on regulated) genes, induced by cold, drought and salt-stress (Stockinger et al., 1997; Liu et al., 1998). In Arabidopsis there are four CBF/DREB1 proteins. Another DRE binding protein, DREB2 (drought responsive element binding factor2); is from a subfamily of AP2/EREBP transcription factors that regulate several drought and salt stress induced genes (Liu et al., 1998). A further transcription factor subfamily, known as ERFs (ethylene responsive element binding factors), bind to GCC box cis-element in the promoters of several pathogenesis related $(P R)$ genes (Singh et al., 2002). Therefore, members of the AP2/EREBP family regulate both biotic and abiotic stress responsive gene expression.

\footnotetext{
${ }^{1}$ The conserved amino acid sequence of WRKY domain is WRKYGQK at its $\mathrm{N}$ terminal end and the first letters of this sequence use to name WRKY domain.

${ }^{2}$ NAC domain consist three different genes such as NAM (no apical meristem), ATAF (Arabidopsis transcription activation factor) and CUC (cup-shaped cotyledon) and first letters of each gene use to name NAC domain.
} 
The bZIP family of transcription factors also functions to control stress responsive gene expression. One type of bZIP family transcription factors, the ABFs (Abscisic acid binding factors) bind ABREs (Abscisic acid responsive elements). These ciselements regulate expression of cold, drought and salt induced genes via ABAdependent signal transduction (Choi et al., 2000). Over-expression of ABF3 (Abscisic acid binding factor3) and ABF4 transcription factors in Arabidopsis alters the expression of stress responsive genes and increases drought tolerance in transgenic plants (Kang et al., 2002). Another bZIP subfamily, the TGA (TGACG motif binding factor)/OBF (ocs element binding factor), bind to the as-1/ocs element of PRl genes (Zhang et al., 1999; Despres et al., 2000). NPR1 (non expresser of PR1), a key component of SA (Salicylic Acid) mediated signaling, interacts with TGA/OBF family proteins upon pathogen infection. This physical interaction is essential to enhance the DNA-binding properties of TGA/OBFs which lead to the induction of $P R$ gene transcripts (Eulgem, 2005).

WRKY transcription factors are unique to plants, and are involved in stress responsive gene expression. Members of the WRKY family show enhanced expression following pathogen infection (Singh et al., 2002). As an example, the expression of WRKYs is essential to activate NPRI activity upon pathogen infection (Yu et al., 2001). WRKY factors interact with two $\mathrm{W}$ boxes in the NPR1 5' UTR upon pathogen infection, activating NPRI transcription. Over-expression of several WRKY genes in Arabidopsis has produced an enhanced resistance to pathogens and altered expression of several stress responsive genes (Singh et al., 2002).

The MYB and bHLH families of transcription factors are also reported to be involved in stress-responsive gene expression. These transcription factors specifically bind to MYC and MYB recognition sites in promoters of ABA responsive genes (Abe et al., 2003). Transgenic Arabidopsis over-expressing $A t M Y C 2$ and $A t M Y B 2$ transcription factors genes showed a higher sensitivity to ABA and altered expression of ABA induced genes (Abe et al., 2003).

The above analysis of current literature shows that, many transcription factors are involved in regulation of stress responsive gene expression. Some of the stress genes are induced by several transcription factors, whilst the same transcription factor may behave differently in response to different stress stimuli. Therefore, it is important to understand the mechanism by which transcription factors are recruited to the promoters of stress induced genes, in order to manipulate the stress resistance of crop plants.

\section{General RNA Polymerase II Transcription Cycle}

The process of synthesis of RNA from DNA is called transcription. This process takes place in the nucleus. The cell initiates transcription by directing the RNA 
polymerase II complex to motifs in the DNA in proximity to the coding region of the target gene, known as the promoter. The most common promoter motif in eukaryotic DNA is the TATA box. Transcription initiates through the formation of a pre-initiation complex (PIC) near the transcriptional start. The PIC includes 6 general transcription factors (GTFs) and the RNA polymerase II enzyme (RNAPII). These GTFs are known as TFIIA, TFIIB, TFIID, TFIIE, TFLLF, TFIIH (Hahn, 2004; Thomas and Chiang, 2006); they work together with RNAPII to specify the transcription initiation site (Thomas and Chiang, 2006).

The PIC usually begins with TFIID binding to the TATA box, followed by the entry of the other GTFs and RNAPII either in a sequential assembly or a preassembled RNAPII holoenzyme (Thomas and Chiang, 2006). Formation of a PIC is sufficient for basal level transcription (Thomas and Chiang, 2006). However, for activatordependent (or regulated) transcription, general co-factors are also required. These include mediator, TAFs (TATA Binding Protein associated factors) and upstream stimulatory activity (USA) derived positive and negative co-factors, and are needed to transmit regulatory signals between gene specific activators and the general transcription machinery (Thomas and Chiang, 2006).

With the completion of formation of PIC, dramatic conformational changes occur in the 11-15 base pairs of DNA surrounding the transcription start (Hahn, 2004). Then transcription begins with the synthesis of the first phosphodiester bond of RNA (Hahn, 2004). In several transcription systems, multiple short RNAs, called abortive products, are synthesized before initiating synthesis of full length transcripts (Hahn, 2004).

After synthesis of about 30 bases of RNA, RNAPII, TFIIB, and TFIIF release their contact with the core promoter and the rest of the transcription machinery enter the transcript elongation phase (Hahn, 2004). Many of the GTFs of PIC remain behind at the promoter in the scaffold complex after release of RNAPII from the PIC complex, and this enables re-initiation of transcription (Hahn, 2004). The scaffold complex accelerates subsequent transcription by bypassing the very slow recruitment of GTFs and formation of PIC at the transcriptional start site (Hahn, 2004).

\section{Mediator Complex}

Mediator is a transcriptional co-activator complex which acts as a bridge to convey DNA-bound transcriptional regulators and enhancers to the general RNA polymerase II transcription machinery (Chadick and Asturias, 2005; Bourbon, 2008). Components of mediator were first identified though biochemical and genetic studies in baker's yeast (Saccharomyces cerevisiae), as subunits linking to the RNA Polymerase II holoenzyme (Flanagan et al, 1991). Mediator complexes were subsequently found in other organisms including higher eukaryotes (Bourbon et al., 2004; Bjorklund and Gustafsson, 2005; Bourbon, 2008). 
Of these, the structure and composition of yeast mediator has been studied in most detail. The yeast complex comprises 26 subunits (Guglielmi et al., 2004), and has been grouped through interactor studies into 4 sub-modules known as the head, middle, tail and kinase (CDK) (Asturias et al., 1999) (Figure 1). In mammalian mediator, 30 subunits have been identified (Bourbon et al., 2004). However, prior to 2007 there were no reports of a plant mediator complex, probably due to the low sequence homology between Arabidopsis and other non-plant eukaryotic mediators (Backstrom et al., 2007).

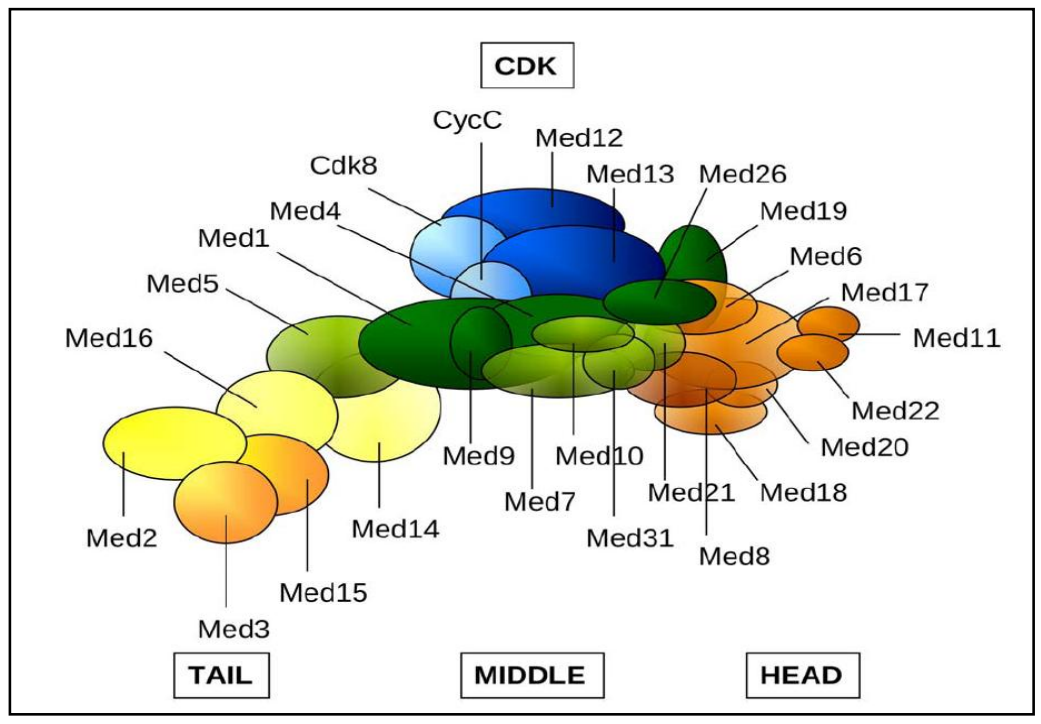

Source: Guglielmi et al. (2004)

Figure 1: Topological organization of yeast mediator

Physical interactions between mediator subunits were revealed in 1992 by Jiang and Stillman, through research on yeast SIN4 (MED16) subunit mutant strain. A mutation in the SIN4 (MED16) subunit of yeast showed loss of function of other tail subunits and therefore, it was suggested that mediator subunits physically interact with each other (Jiang and Stillman, 1992). Consistent with these results, electron microscopic (EM) studies of holoenzyme complexes purified from mutant yeast lacking MED16, showed that the tail mediator modules (MED15, MED3 and MED2) were absent (Chadick and Asturias, 2005). Therefore, they also suggested that the tail portion of mediator formed a subset of physically interacting units. Moreover, the physical association of yeast MED16 and MED14 (Rgr1) proteins were also apparent with the same spectrum of phenotypes observed in sin 4 and rgrl mutant strains (Jiang et al., 1995). 


\section{Mediator in Transcriptional Regulation}

During transcriptional activation and repression, the mediator complex is not directly in contact with gene-specific regulatory sequences (enhancers or silencers). Rather, mediator subunits bind to specific transcriptional activators or repressors, and with other components of PIC (Figure 2) (Bjorklund and Gustafsson, 2005; Bourbon, 2008). Studies have shown that yeast mediator is present both free and in a complex with RNAPII (mediator+RNAPII) (Kim et al., 1994). However, whether the mediator recruit either as free complex or mediator+RNAPII complex to PIC to initiate transcription is not well understood yet.

The CDK module (Sbr8-11 or according to the new nomenclature MED12, MED13, $\mathrm{Cdk} 8$ and $\mathrm{CycC}$ respectively) of mediator has been identified as a negative regulator of transcription. Therefore, mediator complexes with the $\mathrm{CDK}$ module present prevent interaction with RNAPII. Degradation of the CDK module is necessary for mediator to interact with RNAPII and subsequently initiate transcription (Samuelsen et al., 2003) (Figure 2). These same authors proposed a three step model for the degradation of CDK before transcription can commence. First, mediator complexed with CDK binds the transcriptional activation domain, and then to the promoter of the gene; finally at the promoter, the CDK module degrades to facilitate interaction with RNAPII. This means that the mediator attached to PIC comprises only the head, middle and tail modules. Consistent with these results, EM visualization of yeast mediator attached to PIC did not revealed the CDK module (Chadick and Asturias, 2005).

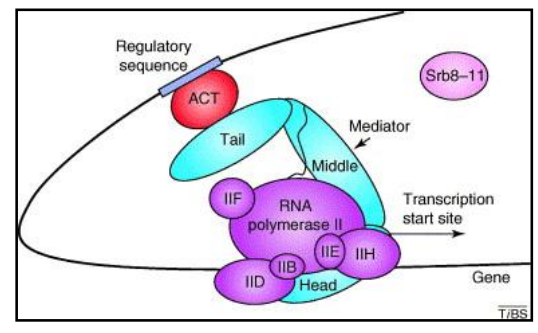

Figure 2a. Transcription activation

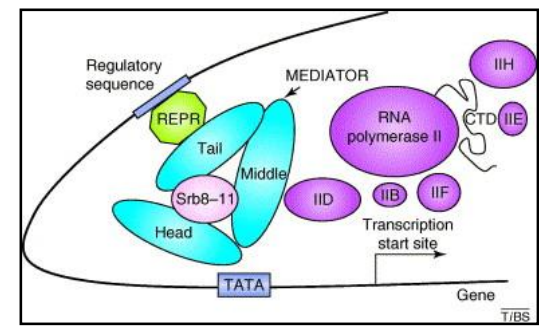

Figure 2b. Transcription repression

Figure 2: A. Mediator functions as a bridge between gene-specific activators and the general polymerase II transcription machinery at the promoter. Activator interactions mainly take place within the tail region of mediator, whereas contacts with polymerase II are localized to the head and middle region. B. Gene specific repressors (REPR) interact with specific mediator subunits and recruit the complex to upstream regulatory DNA sequences. Mediator that is recruited by repressors contains the Srb8-11 (Med12-Med13Cdk8-CycC) module, which prevents interactions with polymerase II and the basal transcription machinery (Source: Bjorklund and Gustafsson, 2005) 
EM images of yeast PIC revealed that the tail domain extends away from RNAPII, though there are multiple physical contacts between RNAPII and the middle and head mediator domains (Chadick and Asturias, 2005). However, large areas of the RNAPII surface $(\sim 75 \%)$ remain available for interaction with other components of the PIC (Chadick and Asturias, 2005). When considering the structural changes of mediator complex during RNAPII interaction, it appears that the structure of the head domain remains same but the tail and middle domains unfold (Chadick and Asturias, 2005).

The carboxyl terminal domain (CTD) of Rpb1 (the largest RNAPII subunit) was identified as necessary for regulation of transcription through mediator (Myers et al., 1998; Chadick and Asturias, 2005). However, more than CTD is required to facilitate the conformational changes necessary for the interaction of mediator with RNAPII (Chadick and Asturias, 2005). In addition, the mediator-RNAPII interaction appears to be at least kingdom-specific, since no mediator+RNAPII complex was formed when yeast mediator was incubated with mammalian RNAPII. This is surprising since the sequences of yeast and mammalian CTDs are highly homologous (Chadick and Asturias, 2005).

\section{Plant Mediator}

In 2007, Backstrom et al. were able to identify plant mediator complexes from Arabidopsis thaliana, using a combination of bioinformatics and biochemical analytical techniques. They found that most of the yeast and metazoan subunits are present in plant mediator, but with a very low sequence homology. Since the Arabidopsis genome encodes $\sim 700$ plant specific transcription factors, to regulate these via mediator, the plant protein recognition motifs would have to differ from those of yeast and other eukaryotes subunits (Backstrom et al., 2007). Their study found homologues for most head and middle domain subunits known from yeast, but very low homology for the candidates of tail domain.

This section of the mediator complex also showed greatest variability when comparing proteins from yeast and metazoans (Backstrom et al., 2007). As mentioned earlier, tail domains make contact with gene-specific enhancers and suppressors of transcriptional regulation. It is likely that the Arabidopsis mediator includes plant specific tail domains (Backstrom et al., 2007). Apart from that they identified potential plant specific mediator subunits named as MED32-37. Backstrom et al. (2007) also identified PFT1 (phytochrome and flowering time; Cerdan and Chory, 2003) as MED25 and SWP (struwwelpeter; Autran et al., 2002) as MED14. These genes were already known as being involved in regulation of flowering time via phyB-dependent pathway ( $P T F 1)$, and defining the duration of cell proliferation $(S W P)$.

Recently Kidd et al. (2009) showed that PFT1 is a key regulator of jasmonate dependent defense in Arabidopsis which is an important defense pathway activate 
upon pathogen infection. Very recently, it has been observed the great involvement of MED16 on various pathways of stress tolerant gene expression such as CBF gene expression on freezing and osmotic stresses and UV and pathogen response gene expression (Knight et al., 2009; Wathugala et al., 2012). However, overexpression of MED16 in Arabidopsis did not increase cold gene expression indicating levels of MED16 alone might not influence activation of target genes (Wathugala et al., 2011). The orthologue of Arabidopsis MED16 has also been identified in rice (Wathugala et al., 2011) and homologues of Arabidopsis MED16 are presented in many agricultural crop species such as maize, papaya, rye etc. 


\section{Conclusions}

It is clear that mediator subunits combine in a complex in equal molar ratios. Moreover, mediator subunits are linked to each other and these links are essential to get maximum output of mediator function on regulation of transcription. Therefore, it can be suggested that the over-expression of one subunit of the mediator complex might not affect the function of the complex as a whole. Therefore, over-expression of one subunit of mediator will not be able to used as a basic molecular tool to improve crop tolerance to stress. However, engineering the protein sequences to improve efficiency and identification of transcription binding sites in mediator sub units will be able to optimizing transcription factor binding and it might lead to improve plant tolerance to environmental stresses. Also, before manipulation of protein sequences of mediator complex proper understanding of the function of mediator subunits on regulation of stress induced gene expression is a must. Therefore, future research on specific roles of individual subunits and of the whole complex will widen our knowledge of the transcriptional regulation of gene expression in plant and will create new routes to improve crop tolerance to multiple environmental stresses.

\section{References}

Abe, H., T. Urao, T. Ito, M. Seki, K. Shinozaki and K. Yamaguchi-Shinozaki (2003). "Arabidopsis AtMYC2 (bHLH) and AtMYB2 (MYB) function as transcriptional activators in abscisic acid signaling" Plant Cell, 15: 63-78.

Autran, D., C. Jonak, K. Belcram, G. T. S. Beemster, J. Kronenberger, O. Grandjean, D. Inze and J. Traas (2002). "Cell numbers and leaf development in Arabidopsis: a functional analysis of the STRUWWELPETER gene" Embo Journal, 21: 60366049 .

Asturias, F.J., Y. W. Jiang, L. C. Myers, C. M. Gustafsson and R.D. Kornberg (1999). "Conserved structures of mediator and RNA polymerase II holoenzyme" Science, 283: $985-987$.

Backstrom, S., N. Elfving, R. Nilsson, G. Wingsle and S. Bjorklund (2007). "Purification of a plant mediator from Arabidopsis thaliana identifies PFT1 as the Med25 subunit" Molecular Cell, 26: 717-729.

Bjorklund, S. and C.M. Gustafsson (2005). "The yeast Mediator complex and its regulation" Trends in Biochemical Sciences, 30: 240-244.

Blokhina, O., E. Virolainen and K. V. Fagerstedt (2003). "Antioxidants, oxidative damage and oxygen deprivation stress: a review" Annals of Botany, 91: 179-194.

Bourbon, H.M. (2008). "Comparative genomics supports a deep evolutionary origin for the large, four-module transcriptional mediator complex" Nucleic Acids Research, 36: 3993-4008. 
Bourbon, H.M., A. Aguilera, A. Z. Ansari, F. J. Asturias, A. J. Berk, S. Bjorklund, T. K. Blackwell, T. Borggrefe, M. Carey, M. Carlson, J. W. Conaway, R. C. Conaway, S. W. Emmons, J. D. Fondell, L. P. Freedman, T. Fukasawa, C. M. Gustafsson, M. Han, X. He, P. K. Herman, A. G. Hinnebusch, S. Holmberg, F. C. Holstege, J. A. Jaehning, Y. J. Kim, L. Kuras, A. Leutz, J. T. Lis, M. Meisterernest, A. M. Naar, K. Nasmyth, J. D. Parvin, M. Ptashne, D. Reinberg, H. Ronne, I. Sadowski, H. Sakurai, M. Sipiczki, P. W. Sternberg, D. J. Stillman, R. Strich, K. Struhl, J. Q. Svejstrup, S. Tuck, F. Winston, R. G. Roeder, R. D. Kornberg (2004). “A unified nomenclature for protein subunits of Mediator complexes linking transcriptional regulators to RNA polymerase II" Molecular Cell, 14: 553-557.

Bjorklund, S. and C. M. Gustafsson (2005). "The yeast Mediator complex and its regulation" Trends in Biochemical Sciences, 30: 240-244.

Cerdan, P. D. and J. Chory (2003). "Regulation of flowering time by light quality" Nature, 423: $881-885$

Chadick, J.Z. and F. J. Asturias (2005). "Structure of eukaryotic Mediator complexes" Trends in Biochemical Sciences, 30: 264-271.

Chen, W.Q., N. J. Provart, J. Glazebrook, F. Katagiri, H. S. Chang, T. Eulgem,F. Mauch, S. Luan, G. Z. Zou, S. A. Whitham, P. R. Budworth, Y. Tao, Z. Y. Xie, X. Chen, S. Lam, J. A. Kreps, J. F. Harper, A. Si-Ammour, B. Mauch-Mani, M. Heinlein, K. Kobayashi, T. Hohn, J. E. Dangl, X. Wang and T. Zhu (2002). "Expression profile matrix of Arabidopsis transcription factor genes suggests their putative functions in response to environmental stresses" Plant Cell, 14: 559-574.

Choi, H.I., J. H. Hong, J. O. Ha, J. Y. Kang and S. Y. Kim (2000). “ABFs, a family of ABAresponsive element binding factors" Journal of Biological Chemistry, 275: 17231730.

Despres, C., C. DeLong, S. Glaze, E. Liu, and P. R. Fobert (2000). "The Arabidopsis NPR1/NIM1 protein enhances the DNA binding activity of a subgroup of the TGA family of bZIP transcription factors" Plant Cell, 12: 279-290.

Eulgem, T. (2005). "Regulation of the Arabidopsis defense transcriptome" Trends in Plant Science, 10: 71-78.

Flanagan, P.M., R. J. Kelleher, M. H. Sayre, H. Tschochner and R. D. Kornberg (1991). “A mediator required for activation of RNA polymerase 11 transcription in vitro" Nature, 350: 436-438.

Fowler, S. and M. F. Thomashow (2002). "Arabidopsis transcriptome profiling indicates that multiple regulatory pathways are activated during cold acclimation in addition to the CBF cold response pathway" Plant Cell, 14: 1675-1690.

Guglielmi, B., N. L. van Berkum, B. Klapholz, T. Bijma, M. Boube, C. Boschiero, H. M. Bourbon, F. C. P. Holstege and M. Werner (2004). "A high resolution protein 
interaction map of the yeast Mediator complex" Nucleic Acids Research, 32: 53795391.

Hahn, S. (2004). "Structure and mechanism of the RNA polymerase II transcription machinery" Nature Structural \& Molecular Biology, 11: 394-403.

Jaglo-Ottosen, K.R., S. J. Gilmour, D. G. Zarka, O. Schabenberger and M. F. Thomashow (1998). "Arabidopsis CBF1 overexpression induces COR genes and enhances freezing tolerance" Science, 280: 104-106.

Jiang, Y.W., P. R. Dohrmann and D. J. Stillman (1995). "Genetic and physical interactions between yeast rgr1 and $\sin 4$ in chromatin organization and transcriptional regulation" Genetics, 140: 47-54.

Jiang, Y.W. and D. J. Stillman (1992). "Involvement of the sin4 global transcriptional regulator in the chromatin structure of saccharomyces-cerevisiae" Molecular and Cellular Biology, 12: 4503-4514.

Jin, H.L., E. Cominelli, P. Bailey, A. Parr, F. Mehrtens, J. Jones, C. Tonelli, B. Weisshaar and C. Martin (2000). "Transcriptional repression by AtMYB4 controls production of UV-protecting sunscreens in Arabidopsis" Embo Journal, 19: 6150-6161.

Kang, J.Y., H. I. Choi, M. Y. Im and S.Y. Kim (2002). "Arabidopsis basic leucine zipper proteins that mediate stress-responsive abscisic acid signaling" Plant Cell, 14: 343357.

Kasuga, M., Q. Liu, S. Miura, , K. Yamaguchi-Shinozaki and K. Shinozaki (1999). "Improving plant drought, salt, and freezing tolerance by gene transfer of a single stress-inducible transcription factor" Nature Biotechnology, 17: 287-291.

Kidd, B.N., C.I. Edgar, K. K. Kumar, E. A. Aitken, P. M. Schenk, J. M. Manners and K. Kazan (2009). "The Mediator Complex Subunit PFT1 Is a Key Regulator of Jasmonate-Dependent Defense in Arabidopsis" Plant Cell, 21: 2237-2252.

Kim, Y.J., S. Bjorklund, Y. Li, M. H. Sayre and R. D. Kornberg (1994). “A multiprotein mediator of transcriptional activation and its interaction with the c-terminal repeat domain of rna-polymerase-II" Cell, 77: 599-608.

Knight H. and M. R. Knight (2001). "Abiotic stress signalling pathways: specificity and cross-talk" Trends in Plant Science, 6: 262-267.

Knight, H., S. G. Mugford, B. Ulker, D. H. Gao, G. Thorlbyand M. R. Knight (2009). "Identification of SFR6, a key component in cold acclimation acting posttranslationally on CBF function" Plant Journal, 58: 97-108.

Kreps, J.A., Y. J. Wu, H. S. Chang, T. Zhu, X. Wang and J. F. Harper (2002). "Transcriptome changes for Arabidopsis in response to salt, osmotic, and cold stress". Plant Physiology, 130: 2129-2141. 
Liu, Q., M. Kasuga, Y. Sakuma, H. Abe, S. Miura, K. Yamaguchi-Shinozaki and K. Shinozaki (1998). "Two transcription factors, DREB1 and DREB2, with an EREBP/AP2 DNA binding domain separate two cellular signal transduction pathways in drought- and low-temperature-responsive gene expression, respectively, in Arabidopsis" Plant Cell, 10: 1391-1406.

Myers, L.C., C. M. Gustafsson, D. A. Bushnell, M. Lui, H. Erdjument-Bromage, P. Tempst and R. D. Kornberg (1998). "The Med proteins of yeast and their function through the RNA polymerase II carboxy-terminal domain" Genes \& Development, 12: 4554.

Nawrath, C., S. Heck, N. Parinthawong and J. P. Metraux (2002). "EDS5, an essential component of salicylic acid-dependent signaling for disease resistance in Arabidopsis, is a member of the MATE transporter family" Plant Cell, 14: 275286.

Riechmann, J.L., J. Heard, G. Martin, L. Reuber, C. Z. Jiang, J. Keddie, L. Adam, O. Pineda, O. J. Ratcliffe, R. R. Samaha, R. Creelman, M. Pilgrim, P. Broun, J. Z. Zhang, D. Ghandehari, B. K. Sherman and C. L. Yu (2000). "Arabidopsis transcription factors: Genome-wide comparative analysis among eukaryotes" Science, 290: 2105-2110.

Rushton, P.J. and I. E. Somssich (1998). "Transcriptional control of plant genes responsive to pathogens" Current Opinion in Plant Biology, 1: 311-315.

Samuelsen, C. O., V. Baraznenok, O. Khorosjutina, H. Spahr, T. Kieselbach, S. Holmberg and C. M. Gustafsson (2003). "TRAP230/ARC240 and TRAP240/ARC250 Mediator subunits are functionally conserved through evolution. Proceedings of the National Academy of Sciences of the United States of America, 100: 6422-6427.

Schenk, P.M., K. Kazan, I. Wilson, J. P. Anderson, T. Richmond, S. C. Somerville and J. M. Manners (2000). "Coordinated plant defense responses in Arabidopsis revealed by microarray analysis" Proceedings of the National Academy of Sciences of the United States of America, 97: 11655-11660.

Seki, M., M. Narusaka, H. Abe, M. Kasuga, K. Yamaguchi-Shinozaki, P. Carninci, Y. Hayashizaki and K. Shinozaki (2001). "Monitoring the expression pattern of 1300 Arabidopsis genes under drought and cold stresses by using a full-length cDNA microarray" Plant Cell, 13: 61-72.

Seki, M., M. Narusaka, J. Ishida, T. Nanjo, M. Fujita, Y. Oono, A. Kamiya, M. Nakajima, A. Enju, T. Sakurai, M. Satou, K. Akiyama, T. Taji, K. Yamaguchi-Shinozaki, P. Carninci, J. Kawai, Y. Hayashizaki and K. Shinozaki (2002). "Monitoring the expression profiles of 7000 Arabidopsis genes under drought, cold and highsalinity stresses using a full-length cDNA microarray" Plant Journal, 31: 279-292.

Shinozaki, K., K. Yamaguchi-Shinozaki and M. Seki (2003). "Regulatory network of gene expression in the drought and cold stress responses" Current Opinion in Plant Biology, 6: 410-417. 
Singh, K.B., R. C. Foley and L. Onate-Sanchez (2002). "Transcription factors in plant defense and stress responses" Current Opinion in Plant Biology, 5: 430-436.

Stockinger, E.J., S. J. Gilmour and M. F. Thomashow (1997). “Arabidopsis thaliana CBF1 encodes an AP2 domain-containing transcriptional activator that binds to the Crepeat/DRE, a cis-acting DNA regulatory element that stimulates transcription in response to low temperature and water deficit" Proceedings of the National Academy of Sciences of the United States of America, 94: 1035-1040.

Thomas, M.C. and C. M. Chiang (2006). "The general transcription machinery and general cofactors" Critical Reviews in Biochemistry and Molecular Biology, 41: 105-178.

Wathugala, D.L., A. Shane, Richards, H. Knight and M. R. Knight (2011). "OsSFR6 is a functional rice orthologue of SENSITIVE TO FREEZING-6 and can act as a regulator of COR gene expression, osmotic stress and freezing tolerance in Arabidopsis" New Phytologist, 191(4): 984-995.

Wathugala, D.L., P. A. Hemsley, C. S. Moffat, P. Cremelie, M. R.Knight and H. Knight (2012). "The Mediator subunit SFR6MED16 controls defense gene expression mediated by salicylic acid and jasmonate responsive pathways" New Phytologist, 195: 217-230.

Xiong, L.M., K. S. Schumaker and J. K. Zhu (2002). "Cell signaling during cold, drought, and salt stress" Plant Cell, 14: 165-183.

Yu, D.Q., C. H. Chen and Z. X. Chen (2001). "Evidence for an important role of WRKY DNA binding proteins in the regulation of NPR1 gene expression" Plant Cell, 13: 1527-1539.

Zhang, Y.L., W. H. Fan, M. Kinkema, X. Li and X. N. Dong (1999). "Interaction of NPR1 with basic leucine zipper protein transcription factors that bind sequences required for salicylic acid induction of the PR-1 gene" Proceedings of the National Academy of Sciences of the United States of America, 96: 6523-6528. 